\title{
Strong coexistence for a model with endogenous evolution of heterogeneous agents
}

\author{
Ahmad Naimzada ${ }^{a *}$, Marina Pireddu ${ }^{\mathrm{b} \dagger}$ \\ ${ }^{a}$ Dept. of Economics, Management and Statistics, University of Milano-Bicocca, \\ U6 Building, Piazza dell'Ateneo Nuovo 1, 20126 Milano, Italy. \\ ${ }^{\mathrm{b}}$ Dept. of Mathematics and Applications, University of Milano-Bicocca, \\ U5 Building, Via Cozzi 55, 20125 Milano, Italy.
}

\begin{abstract}
We propose two exchange economy evolutionary models with heterogeneous agents, in which the share updating mechanisms depend on the goods' consumption, described in terms of the calorie intakes. In the first setting we assume that the share updating rule is monotone in the calorie intake, while in the second framework we suppose that it is non-monotone. In both scenarios we investigate whether there may be multistability phenomena involving nontrivial market stationary equilibria at which the calorie intakes for the two groups of agents differ. The answer is negative in the first setting, while it is affirmative in the second framework.
\end{abstract}

Keywords: Endogenous preferences; multistability; weak and strong coexistence; bifurcation analysis.

\footnotetext{
*Tel.: $\quad+390264485813 ; \quad$ fax: $\quad+390264483085 . \quad$ E-mail address: ahmad.naimzada@unimib.it

${ }^{\dagger}$ Corresponding author. Tel.: +39 0264485767; fax: +390264485705 . E-mail address: marina.pireddu@unimib.it
} 


\section{Introduction}

In the present paper we aim to let emerge the richness of asymptotic dynamic behaviors hidden inside the model proposed in [1] and further developed in [2]. Indeed, in order to explore the variety of asymptotic outcomes intrinsic in those works, we here relax some too restrictive assumptions made therein. More precisely, we reconsider the exchange economy evolutionary models with agents heterogeneous in the structure of preferences, in which the weights assigned to the two consumption goods in the Cobb-Douglas utility functions do not coincide across groups, dealt with in $[1,2]$, by allowing the endowments of the two goods to differ across groups of agents, in agreement with the possible extensions proposed in [1]. Moreover, like in [1, 2], we assume that the growth rate of each population group is determined by a biological payoff function, which depends on the consumption of the group's agents, described in terms of the assumed calorie intake. However, we here consider two functional forms for the share updating mechanism, and we investigate how the different modeling choices influence the number of steady states and their features. Indeed, in the first model we analyze, like in [1], the rule according to which shares are updated is monotonically increasing in the calorie intake, while in the second framework we study, as in [2], we replace the monotone population growth rate assumed in [1] with a bell-shaped map, increasing with the calorie intake up to a certain threshold value, above which it becomes decreasing.

The need to relax some of the hypotheses made in $[1,2]$ comes from the following simple observation. In both the frameworks in $[1,2]$, under the endowment homogeneity assumption, at the unique nontrivial (i.e., characterized by the coexistence between the two groups of agents) locally stable equilibrium the calorie intakes for the two groups coincide, albeit the heterogeneity in the structure of preferences. We will refer to such feature as to weak coexistence, while we will talk of strong coexistence in those cases in which there exist nontrivial locally stable equilibria at which the calorie intakes for the two groups of agents differ. Since the weak coexistence detected in $[1,2]$ is in deep disagreement with the empirical outcomes and limits the interpretative value of those settings, we here allow for endowment heterogeneity to try to fix the issue. In particular, in our investigation we adopt a bifurcation analysis approach.

We stress that both the share updating mechanisms we consider are empirically grounded. Namely, according to [1], a monotone population growth 
rate is suitable to represent the long-run centuries-old trend, as the diet of a population group affects its long-term survival. On the other hand, biological payoff functions monotonically increasing in the calorie intake well describe food regimes characterized by a calorie shortage, but they are not appropriate to represent the framework of contemporary developed countries and the negative effects of overconsumption on health and survival, which are instead properly described by bell-shaped maps. Indeed, as argued in [3], monotone survival functions do not fit aggregate data and a broad epidemiological literature (see e.g. $[4,5,6,7]$ ) has shown a non-monotone relationship between the corpulence and mortality risks. Such non-monotone relationship is clearly represented by Waaler's $U$-shaped curves in [8], commented from an intertemporal viewpoint in [9]. The relevance of those findings gave rise to the so-called "economics of obesity" (cf. the survey [10]). Hence, the second setting we deal with, as the one in [2], is suitable to represent the long-run centuries-old trend from the industrial revolution on, while the first framework we consider, like that in [1], is well-suited to describe the long-run centuries-old trend before the industrial revolution.

We recall that evolutionary frameworks with binary choices at a collective level have been considered, for instance, in [11, 12, 13, 14]. On the other hand, differently from those papers, the binary choice here occurs between preference structures that characterize the two groups of agents, which are embedded in a general equilibrium framework, where a price mechanism operates.

As regards the findings obtained in $[1,2]$, we recall that both settings result in one-dimensional continuous-time dynamical systems. More precisely, the analysis in those papers concerns the existence and local stability of trivial and nontrivial market stationary equilibria. In [1] at most one nontrivial market stationary equilibrium, which when exists is stable, and two trivial equilibria are found. Moreover, by construction, at the nontrivial market stationary equilibrium the calorie intakes coincide across groups. In [2], in addition to the three equilibria in [1], (up to) two additional nontrivial market stationary equilibria are detected. Furthermore, the (possibly existing) nontrivial equilibrium found in [1] may become unstable in the context considered in [2], and also the two trivial equilibria may have different dynamic behaviors with respect to [1]. In particular, unlike the framework in [1], the setting in [2] displays multistability phenomena, characterized by the presence of multiple, trivial and nontrivial, locally stable market stationary equilibria. 
We remark that, as explained in [2], multistability may be considered as a source of richness for the framework under analysis because, other parameters being equal, i.e., under the same institutional, cultural and social conditions, it allows to explain different historical trajectories and evolutionary paths. The initial conditions, leading to the various attractors, represent indeed a summary of the past history, which in the presence of multistability phenomena does matter in determining the evolution of the system. Such property, in the literature on complex systems, is also called "path-dependence" (see [15]). Moreover, in the specific context we deal with, the presence of multiple equilibria well represents the variety of historical experiences across different countries in relation to the approach they adopt towards food, diet and consequently towards obesity (consider e.g., according to [10], the different scenarios in the U.S. and in the Mediterranean countries).

On the other hand, the multistability phenomena detected in [2] are not fully satisfying from an interpretative viewpoint, as at the only involved nontrivial market stationary equilibrium the calorie intakes for both groups of agents coincide, i.e., there is just weak coexistence.

For such reason, in the present paper we add to the frameworks considered in $[1,2]$ the heterogeneity assumption for endowments, and we study market equilibria, which link equilibrium price and optimal consumption quantities to population shares, in view of investigating whether that new heterogeneity hypothesis may generate multistability phenomena involving nontrivial market stationary equilibria with strong coexistence, at which the calorie intakes for the two groups of agents differ. As we will see, we find that the answer is negative in the setting proposed in [1], while it is affirmative in the framework in [2]. Indeed, in the former context, even under the endowment heterogeneity assumption, we do not observe multistability phenomena, since the possible outcomes are those detected in [1] and recalled above. In the latter framework, in addition to the results obtained in [2], we find instead multistability phenomena in which the nontrivial equilibrium is characterized by different calorie intakes for the two groups of agents. We stress that this is a crucial difference between the homogeneous and heterogeneous endowment settings, because in case of strong coexistence the involved nontrivial equilibrium displays a deeper degree of heterogeneity between groups, not only in terms of population shares, but also from a caloric viewpoint.

Finally, we perform a bifurcation analysis in order to understand how the market stationary equilibria with different calorie intakes do emerge. We obtain a qualitative result, showing that, according to the relative endowment 
values, those equilibria can appear through a transcritical or a saddle-node bifurcations. Our analysis allows us to discuss the local stability and the basins of attraction of the stationary equilibria that emerge through such bifurcations.

The remainder of the paper is organized as follows. In Section 2 we present the general model. In Sections 3 and 4 we respectively describe the settings with monotone and non-monotone population growth rates. In Section 5 we perform a bifurcation analysis, showing the possible dynamic scenarios and, in particular, those characterized by the presence of strong coexistence. In Section 6 we briefly discuss our results and propose some possible extensions of our models.

\section{The model}

We start our discussion recalling the framework with homogeneous endowments in [1], where the authors consider a continuous-time model describing an exchange economy with a continuum of agents, which may be of type $\alpha$ or of type $\beta$. There are two consumption goods, $x$ and $y$, and agent preferences are described by Cobb-Douglas utility functions, i.e., $U_{i}(x, y)=x^{i} y^{1-i}$, for $i \in\{\alpha, \beta\}$, with $0<\beta<\alpha<1$. Both kinds of agents have the same endowments of the two goods, denoted respectively by $w_{x}$ and $w_{y}$. The analysis is performed in terms of the relative price $p(t)=p_{y}(t) / p_{x}(t)$, where $p_{x}(t)$ and $p_{y}(t)$ are the prices at time $t$ for goods $x$ and $y$, respectively. The size of the population of kind $\alpha(\beta)$ at time $t$ is denoted by $A(t)(B(t))$ and the normalized variable $a(t)=(A(t)) /(A(t)+B(t))$ represents the fraction of the population composed by the agents of type $\alpha$.

We now present the definition of market equilibrium, we will refer to in the remainder of the paper.

Definition 2.1 Given the economy and the population share a $(t)$, a market equilibrium at time $t$ is a vector $\left(p^{*}(t), x_{i}^{*}(t), y_{i}^{*}(t)\right)$, with $i \in\{\alpha, \beta\}$, such that:

- every kind of agent chooses a utility-maximizing consumption bundle, given $p^{*}(t)$;

- the markets for the two goods clear. 
The market equilibrium price at time $t$ is then given by

$$
p^{*}(t)=\frac{[1-(a(t) \alpha+(1-a(t)) \beta)] w_{x}}{(a(t) \alpha+(1-a(t)) \beta) w_{y}}
$$

and the consumer equilibrium quantities of the two goods for an agent of type $i \in\{\alpha, \beta\}$ are

$$
\begin{aligned}
& x_{i}^{*}(t)=i\left(w_{x}+p^{*}(t) w_{y}\right)=\frac{i w_{x}}{a(t) \alpha+(1-a(t)) \beta}, \\
& y_{i}^{*}(t)=(1-i)\left(\frac{w_{x}}{p^{*}(t)}+w_{y}\right)=\frac{(1-i) w_{y}}{1-(a(t) \alpha+(1-a(t)) \beta)} .
\end{aligned}
$$

The framework we are going to analyze generalizes the one recalled above, as we relax the homogeneity assumption for endowments across groups. Hence, we introduce the endowments of good $x$ for the agents of type $\alpha$ and $\beta$, denoted respectively by $w_{x, \alpha}$ and $w_{x, \beta}$, and similarly we will denote by $w_{y, \alpha}$ and $w_{y, \beta}$ the endowments of good $y$ for the agents of type $\alpha$ and $\beta$, respectively. Recalling Definition 2.1, we have the following result.

Proposition 2.1 Given the economy with heterogeneous endowment distribution and $a(t)$, the market equilibrium price at time $t$ is

$$
p^{*}(t)=\frac{a(t)(1-\alpha) w_{x, \alpha}+(1-a(t))(1-\beta) w_{x, \beta}}{a(t) \alpha w_{y, \alpha}+(1-a(t)) \beta w_{y, \beta}}
$$

and the consumer equilibrium quantities of the two goods for an agent of type $i \in\{\alpha, \beta\}$, compatible with the market equilibrium, are

$$
x_{i}^{*}(t)=i\left(w_{x, i}+p^{*}(t) w_{y, i}\right), \quad y_{i}^{*}(t)=(1-i)\left(\frac{w_{x, i}}{p^{*}(t)}+w_{y, i}\right),
$$

i.e.,

$$
\begin{aligned}
& x_{\alpha}^{*}(t)=\alpha\left(\frac{a(t) w_{x, \alpha} w_{y, \alpha}+(1-a(t)) \beta w_{x, \alpha} w_{y, \beta}+(1-a(t))(1-\beta) w_{x, \beta} w_{y, \alpha}}{a(t) \alpha w_{y, \alpha}+(1-a(t)) \beta w_{y, \beta}}\right), \\
& x_{\beta}^{*}(t)=\beta\left(\frac{(1-a(t)) w_{x, \beta} w_{y, \beta}+a(t)(1-\alpha) w_{x, \alpha} w_{y, \beta}+a(t) \alpha w_{x, \beta} w_{y, \alpha}}{a(t) \alpha w_{y, \alpha}+(1-a(t)) \beta w_{y, \beta}}\right), \\
& y_{\alpha}^{*}(t)=(1-\alpha)\left(\frac{a(t) w_{x, \alpha} w_{y, \alpha}+(1-a(t)) \beta w_{x, \alpha} w_{y, \beta}+(1-a(t))(1-\beta) w_{x, \beta} w_{y, \alpha}}{a(t)(1-\alpha) w_{x, \alpha}+(1-a(t))(1-\beta) w_{x, \beta}}\right), \\
& y_{\beta}^{*}(t)=(1-\beta)\left(\frac{(1-a(t)) w_{x, \beta} w_{y, \beta}+a(t)(1-\alpha) w_{x, \alpha} w_{y, \beta}+a(t) \alpha w_{x, \beta} w_{y, \alpha}}{a(t)(1-\alpha) w_{x, \alpha}+(1-a(t))(1-\beta) w_{x, \beta}}\right) .
\end{aligned}
$$


Proof. The desired conclusions follow by simple computations, solving the consumer maximization problems for agents of types $\alpha$ and $\beta$ and using the market clearing condition for one of the two goods.

Once we specify a dynamical rule for the population share evolution, it is also possible to give the definition of market stationary equilibrium, for both the homogeneous and heterogeneous endowment contexts.

Definition 2.2 Given the economy, the vector $\left(a^{*}, p^{*}, x_{i}^{*}, y_{i}^{*}\right), i \in\{\alpha, \beta\}$, is a market stationary equilibrium if $a^{*}$ is constant and if, given $a^{*},\left(p^{*}, x_{i}^{*}, y_{i}^{*}\right)$, $i \in\{\alpha, \beta\}$, is a market equilibrium for every $t$.

For the sake of brevity, in what follows we will identify market stationary equilibria just with the population share $a$, since it determines all other equilibrium components, which will be constant as well.

\section{The monotone population growth rate set- ting}

According to [1], the calorie intake $K_{i}(t)$ of an agent of type $i \in\{\alpha, \beta\}$ at time $t$ is given by a linear combination of the units $x_{i}(t)$ and $y_{i}(t)$ of goods $x$ and $y$ he consumes, weighted respectively with the calories that each agent derives from the consumption of a unit of good $x$ and of good $y$, i.e., $K_{i}(t)=c_{x} x_{i}(t)+c_{y} y_{i}(t)$. In that framework, denoting by $\bar{K}$ the calorie subsistence level, the growth rate of the population of type $i$ is assumed to be

$$
K_{i}(t)-\bar{K}
$$

so that the evolution of the two groups of consumers is described by the following system

$$
\left\{\begin{array}{l}
\frac{d A(t)}{d t}=\left(K_{\alpha}(t)-\bar{K}\right) A(t) \\
\frac{d B(t)}{d t}=\left(K_{\beta}(t)-\bar{K}\right) B(t)
\end{array}\right.
$$

which, in terms of the normalized variable $a(t)$, becomes equivalent to

$$
\frac{d a(t)}{d t}=a(t)(1-a(t))\left(K_{\alpha}(t)-K_{\beta}(t)\right) .
$$


Since in the homogeneous endowment setting considered in [1]

$$
K_{\alpha}(t)-K_{\beta}(t)=(\alpha-\beta)\left(\frac{c_{x} w_{x}}{a(t) \alpha+(1-a(t)) \beta}-\frac{c_{y} w_{y}}{1-a(t) \alpha-(1-a(t)) \beta}\right),
$$

(3.2) can be rewritten as

$$
\frac{d a(t)}{d t}=(\alpha-\beta) a(t)(1-a(t))\left(\frac{c_{x} w_{x}}{a(t) \alpha+(1-a(t)) \beta}-\frac{c_{y} w_{y}}{1-a(t) \alpha-(1-a(t)) \beta}\right) .
$$

Notice that $\bar{K}$ does not affect the one-dimensional differential equation.

Recalling Definition 2.2, market stationary equilibria will be called trivial if they are not characterized by the coexistence between the two groups of agents, and nontrivial otherwise. In addition to the trivial market stationary equilibria $a=0$ and $a=1$, a nontrivial market stationary equilibrium is given by $a=a^{*}$, with

$$
a^{*}=\frac{(1-\beta) c_{x} w_{x}-\beta c_{y} w_{y}}{(\alpha-\beta)\left(c_{x} w_{x}+c_{y} w_{y}\right)}
$$

as long as $a^{*} \in(0,1)$, i.e., for $c_{x} w_{x} \in\left(\left(\beta c_{y} w_{y}\right) /(1-\beta),\left(\alpha c_{y} w_{y}\right) /(1-\alpha)\right)$. Such market stationary equilibrium, when it exists, is always stable for the model considered in [1]. In that paper no comments are made on the local stability of the dynamical system at $a=0$ and $a=1$. However, a simple continuity argument shows that, when $a^{*} \in(0,1)$, then $a=0$ and $a=1$ are always unstable. When instead $a^{*} \notin(0,1), a=0$ may be unstable and $a=1$ stable, or vice versa.

We stress that, by construction, at $a=a^{*}$ it necessarily holds that $K_{\alpha}(t)=$ $K_{\beta}(t)$, for every $t$, and thus such equilibrium, although nontrivial, is not characterized by a strong coexistence between groups, i.e., by an heterogeneity in terms of calorie intakes.

When allowing for heterogeneous endowments, and thus employing the equilibrium expressions found in Proposition 2.1, the resulting formulation for

$$
K_{\alpha}(t)-K_{\beta}(t)=c_{x}\left(x_{\alpha}^{*}(t)-x_{\beta}^{*}(t)\right)+c_{y}\left(y_{\alpha}^{*}(t)-y_{\beta}^{*}(t)\right)
$$

is much more complicated than the one in (3.3) and we do not report it for sake of brevity.

In the next result, we find the market stationary equilibria for (3.2) in the more general context. 
Proposition 3.1 Given the economy, the market stationary equilibria for (3.2) with heterogeneous endowments are $a=0, a=1$, and up to two nontrivial ones in $(0,1)$.

Proof. Inserting (3.5) in (3.2) and setting $d a(t) / d t=0$, the solutions are given by $a=0, a=1$, plus the roots of $K_{\alpha}(t)-K_{\beta}(t)=0$. Since the latter is a quadratic equation, it admits up to two solutions in $(0,1)$, corresponding to the nontrivial market stationary equilibria.

Several numerical simulations we performed suggest that, like for the framework considered in [1], at most one of the two nontrivial market stationary equilibria found in Proposition 3.1, we denote by $a=a^{*}$ in analogy with $(3.4)$, lies in $(0,1)$. When $a^{*}$ belongs to $(0,1)$, it is always stable, while $a=0$ and $a=1$ are always unstable. When instead $a^{*}$ does not belong to $(0,1)$, then $a=0$ may be unstable and $a=1$ stable, or vice versa (see the red graph in Figure 1 (A), (B) and (I)).

Hence, it seems that, even relaxing the homogeneity assumption on the endowments in the monotone population growth rate framework considered in [1], no coexistence phenomena may arise. Moreover, by construction, at the nontrivial market stationary equilibria it holds that $K_{\alpha}(t)=K_{\beta}(t)$, for every $t$, and thus they do not allow for a strong coexistence between groups.

\section{The non-monotone population growth rate setting}

In the present section we recall the framework with homogeneous endowments analyzed in [2] and we explain how it changes when endowments may differ across groups.

In such context, instead of dealing with the monotone growth rate in (3.1), it is assumed, in agreement with the empirical literature, the existence for the growth rate of a threshold value, above which an increasing calorie intake becomes harmful, rather than beneficial. In symbols, the growth rate is given by

$$
\frac{1}{1+\sigma\left(K_{i}(t)-\widehat{K}\right)^{2}}
$$

where $\sigma$ is a positive parameter representing the intensity of the decrease in the growth rate due to an increase in the distance between the calorie intake 
$K_{i}(t)$ and the threshold value $\widehat{K}$. In this manner $\widehat{K}$ is no more interpretable as the calorie subsistence level $\bar{K}$ in (3.1), but as the desirable calorie intake, which allows maximizing the growth rate. With such modification, the evolution of the two groups of consumers is described by the following system

$$
\left\{\begin{aligned}
\frac{d A(t)}{d t} & =\frac{A(t)}{1+\sigma\left(K_{\alpha}(t)-\widehat{K}\right)^{2}} \\
\frac{d B(t)}{d t} & =\frac{B(t)}{1+\sigma\left(K_{\beta}(t)-\widehat{K}\right)^{2}}
\end{aligned}\right.
$$

which, introducing the population fraction $a(t)$, becomes equivalent to

$$
\begin{aligned}
\frac{d a(t)}{d t} & =a(t)(1-a(t))\left(\frac{1}{1+\sigma\left(K_{\alpha}(t)-\widehat{K}\right)^{2}}-\frac{1}{1+\sigma\left(K_{\beta}(t)-\widehat{K}\right)^{2}}\right)= \\
& =a(t)(1-a(t))\left(K_{\alpha}(t)-K_{\beta}(t)\right)\left(\frac{\sigma\left(2 \widehat{K}-K_{\alpha}(t)-K_{\beta}(t)\right)}{\left(1+\sigma\left(K_{\alpha}(t)-\widehat{K}\right)^{2}\right)\left(1+\sigma\left(K_{\beta}(t)-\widehat{K}\right)^{2}\right)}\right) .
\end{aligned}
$$

If endowments are homogeneous across groups, i.e., in the context considered in [2], the expression for $K_{\alpha}(t)-K_{\beta}(t)$ is given by (3.3). In this case, in addition to the trivial market stationary equilibria $a=0$ and $a=1$, there are up to three nontrivial equilibria. One of them comes from the condition $K_{\alpha}(t)=K_{\beta}(t)$ and coincides with $a=a^{*}$ in (3.4), while the other two nontrivial equilibria derive from the condition $K_{\alpha}(t)+K_{\beta}(t)=2 \widehat{K}$. The (possibly existing) nontrivial equilibrium found in [1] may become unstable in the context considered in [2], and also the trivial equilibria may have different dynamic behaviors in the two settings. Moreover, unlike the framework in [1], the setting in [2] displays multistability phenomena, characterized by the presence of multiple, trivial and nontrivial, locally stable market stationary equilibria. On the other hand, in the multistability phenomena detected in [2] at the only involved nontrivial market stationary equilibrium the calorie intakes for both groups of agents coincide, and thus there is just weak coexistence.

When relaxing the homogeneity assumption on endowments, the expression for $K_{\alpha}(t)-K_{\beta}(t)$ is given by (3.5).

In the next result, we find the market stationary equilibria for (4.1) with heterogeneous endowments.

Proposition 4.1 Given the economy, the market stationary equilibria for (4.1) with heterogeneous endowments are $a=0, a=1$, and up to four nontrivial ones in $(0,1)$. 
Proof. Inserting (3.5) in (4.1) and setting $d a(t) / d t=0$, the solutions are given by $a=0, a=1$, plus the roots of $K_{\alpha}(t)-K_{\beta}(t)=0$ and of $K_{\alpha}(t)+$ $K_{\beta}(t)=2 \widehat{K}$. Since both $K_{\alpha}(t)-K_{\beta}(t)=0$ and $K_{\alpha}(t)+K_{\beta}(t)=2 \widehat{K}$ are quadratic equations, they admit up to two solutions in $(0,1)$ each, corresponding to the nontrivial market stationary equilibria for (4.1) with heterogeneous endowments.

We stress that the market stationary equilibria coming from the condition $K_{\alpha}(t)=K_{\beta}(t)$ coincide with those already discussed at the end of Section 3. In particular, it seems that at most one of them can lie in $(0,1)$ and we still denote it by $a=a^{*}$. We will call $a=a_{1}^{*}$ and $a=a_{2}^{*}$, with $a_{1}^{*} \leq a_{2}^{*}$, the other two nontrivial equilibria, deriving from the condition $K_{\alpha}(t)+K_{\beta}(t)=2 \widehat{K}$. These are the most interesting ones from an interpretative viewpoint, being characterized by strong coexistence, as the calorie intakes for the two groups differ. Furthermore, like we will see in Figure 1 (F), both of them can lie in $(0,1)$, even if by a simple continuity argument at most one of them can be stable. Of course this is true when no equilibria with $K_{\alpha}(t)=K_{\beta}(t)$ belong to $(0,1)$. On the other hand, when an equilibrium with $K_{\alpha}(t)=K_{\beta}(t)$ belongs to $(0,1)$, the latter seems to be always stable, and thus it is not possible that both the nontrivial equilibria satisfying the condition $K_{\alpha}(t)+K_{\beta}(t)=2 \widehat{K}$ are stable (cf. Figure $1(\mathrm{~F})$ ).

In the next section, we will illustrate the results described so far, as well as perform a bifurcation analysis, which shows the possible outcomes obtainable when moving, for instance, parameter $w_{y, \alpha}$, i.e., one of the newly introduced endowment values. In particular, we will find that the market stationary equilibria with different calorie intakes can emerge through a transcritical or a saddle-node bifurcations. We will discuss qualitatively their appearance, fixing the value of all parameters, but $w_{y, \alpha}$.

\section{Bifurcation analysis and possible scenarios}

In view of the next investigation, it is expedient to introduce the one-dimensional maps $f, g:[0,1] \rightarrow \mathbb{R}$ related to (3.2) and (4.1), respectively, in the heterogeneous endowments setting and defined as

$$
\begin{aligned}
& f(a)=a(1-a)\left(K_{\alpha}-K_{\beta}\right) \\
& g(a)=a(1-a)\left(K_{\alpha}-K_{\beta}\right)\left(\frac{\sigma\left(2 \widehat{K}-K_{\alpha}-K_{\beta}\right)}{\left(1+\sigma\left(K_{\alpha}-\widehat{K}\right)^{2}\right)\left(1+\sigma\left(K_{\beta}-\widehat{K}\right)^{2}\right)}\right),
\end{aligned}
$$


with $K_{\alpha}-K_{\beta}$ like in (3.5).

Let us first focus on the monotone population growth rate setting. As already recalled in Section 3, in the homogeneous endowments framework in [1], when $a^{*} \in(0,1)$, then $a=0$ and $a=1$ are always unstable; when instead $a^{*} \notin(0,1), a=0$ may be unstable and $a=1$ stable, or vice versa. Hence, in that setting no multistability phenomena, characterized by the presence of multiple locally stable market stationary equilibria, may arise. The same conclusions seem to hold also in the case of heterogeneous endowments. The corresponding scenarios can be found, for instance, in Figure 1 (A), (B) and (I), where we draw the graph of $f$ in red (and of $g$ in blue) for $c_{x}=1.5, w_{x, \beta}=0.5, c_{y}=1.3, \alpha=0.7, \beta=0.3$ (as well as for $\widehat{K}=2$ and $\sigma=0.9$ for $g$ ) and we make the other endowment values vary as follows: $w_{x, \alpha}=0.4, w_{y, \alpha}=0.1, w_{y, \beta}=0.3$ in $(\mathrm{A}), w_{x, \alpha}=0.4, w_{y, \alpha}=0.1, w_{y, \beta}=0.2$ in (B), and $w_{x, \alpha}=0.8, w_{y, \alpha}=2.5, w_{y, \beta}=0.2$ in (I). Because of the next discussion, we also report the endowment values in the remaining frameworks considered in Figure 1, i.e., $w_{x, \alpha}=0.4, w_{y, \beta}=0.2$, and $w_{y, \alpha}=0.7$ in $(\mathrm{C})$, $w_{y, \alpha}=1$ in (D), $w_{y, \alpha}=1.5005$ in $(\mathrm{E}), w_{y, \alpha}=1.95$ in $(\mathrm{F}), w_{y, \alpha}=2.0846$ in $(\mathrm{G})$ and $w_{y, \alpha}=2.5$ in $(\mathrm{H})$.

Turning now to the non-monotone growth rate framework, we observe that, both in the homogeneous and heterogeneous endowments settings, in addition to reproducing all the scenarios arising from the context in [1], it is also possible to find multistability phenomena, involving trivial and nontrivial equilibria. For the homogeneous endowment setting, we refer the reader to [2], while for the heterogeneous endowments framework, see the graph of $g$ in blue in Figure 1, where in (A) and (B) we reproduce the scenarios in [1], ${ }^{1}$ while in $(\mathrm{C})-(\mathrm{F})$ we obtain multistability phenomena, involving both trivial and nontrivial equilibria.

We now better study the mutual relationship between the stability of the equilibria in the monotone and non-monotone growth rate frameworks with heterogeneous endowments, performing a qualitative bifurcation analysis. In particular, we investigate the emergence/disappearance and stability gain/loss of equilibria on varying the value of endowment $w_{y, \alpha}$.

Figure 1 shows that for small values of $w_{y, \alpha}$ maps $f$ and $g$ are analogous

\footnotetext{
${ }^{1}$ It is easy to find parameter values which generate the scenario symmetric to (A) with respect to the $x$-axis, with both $f$ and $g$ positive on $(0,1)$. A possible such choice is given by $c_{x}=0.5, w_{x, \alpha}=2, w_{x, \beta}=0.5, c_{y}=1.3, w_{y, \alpha}=0.4, w_{y, \beta}=0.3, \alpha=0.5, \beta=0.4, \widehat{K}=2$ and $\sigma=0.9$.
} 


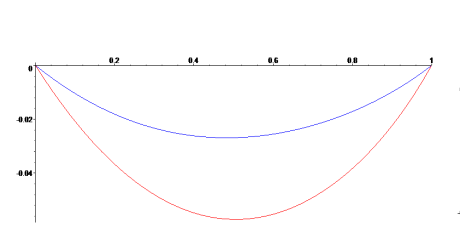

(A)

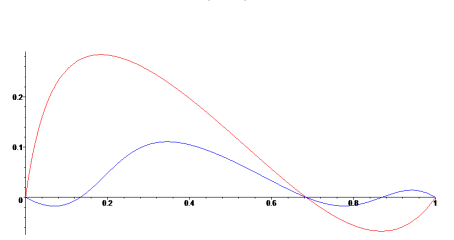

(D)

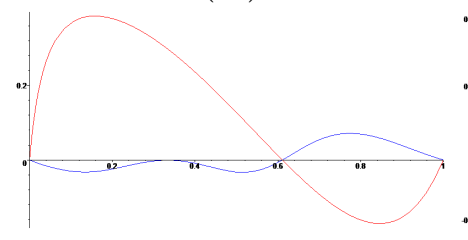

(G)

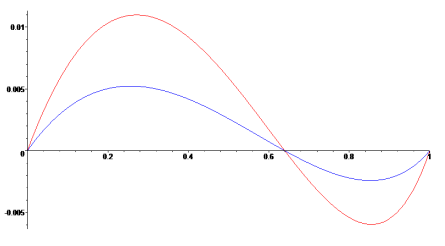

(B)

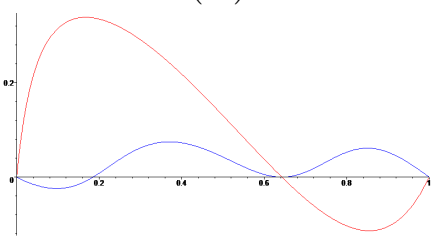

(E)

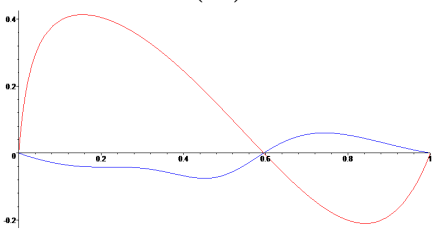

( $\mathrm{H})$

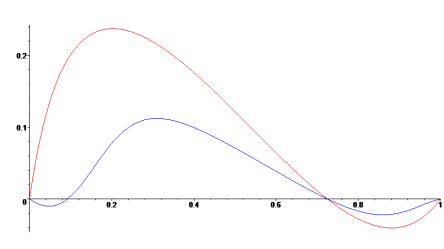

(C)

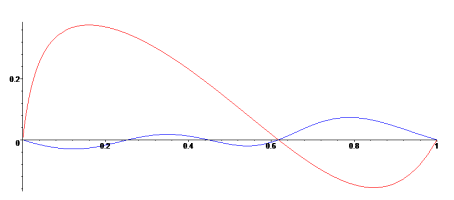

$(\mathrm{F})$

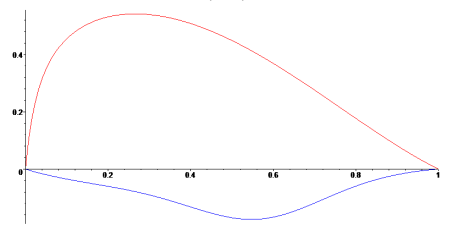

(I)

Figure 1: The graphs of the first iterate of $f$ (in red) and of $g$ (in blue) for different endowment values.

from a stability viewpoint (see (A) and (B) for $w_{y, \alpha}=0.1$ ), and thus we find for both the monotone and non-monotone growth rate frameworks the same dynamic behaviors as in [1], while for large values of $w_{y, \alpha}$ maps $f$ and $g$ display an opposite behavior (see (H) and (I) for $w_{y, \alpha}=2.5$ ); for intermediate increasing values of $w_{y, \alpha}$, the behaviors of $f$ and $g$ become less and less similar (see (C) for $w_{y, \alpha}=0.7 \operatorname{through}(\mathrm{G})$ for $w_{y, \alpha}=2.09$ ).

As concerns multistability, we notice that the locally stable equilibria for $g$ are $a=0, a=a^{*}$ in (C); $a=0, a=a^{*}, a=1$ in (D); $a=0, a=1$ in (E), $(\mathrm{G})$ and $(\mathrm{H}) ; a=0, a=a_{2}^{*}, a=1$ in $(\mathrm{F})$. The unstable equilibria in (C)$(\mathrm{H})$ play the role of separating the basins of attraction of the locally stable equilibria: trajectories will be attracted by one or the other of the various locally stable equilibria according to the chosen initial condition. In regard to (A) and (B), since there the monotone and non-monotone growth rate frameworks are dynamically equivalent to the setting in [1], no multistability 
phenomena may arise and indeed the unique stable equilibrium we find in (A) is given by $a=0$, while in (B) is given by $a=a^{*}{ }^{2}$

Hence, like for the framework considered in [2], also when introducing heterogeneous endowments at most one of the multiple locally stable equilibria is characterized by the coexistence between the two groups of agents, ${ }^{3}$ while in the remaining one(s) just a group survives. On the other hand, differently from [2], we find scenarios like that in Figure $1(\mathrm{~F})$ in which at such nontrivial equilibrium there is strong coexistence, as the calorie intakes for the two groups of agents differ. For instance at $a=a_{2}^{*}=0.4474$ in Figure $1(\mathrm{~F})$ we have $K_{\alpha}=2.372717260$ and $K_{\beta}=1.627282742$. We stress that this is a crucial difference between the homogeneous and heterogeneous endowments settings, as a nontrivial equilibrium with $K_{\alpha} \neq K_{\beta}$ displays a deeper degree of heterogeneity between groups, not only in terms of population shares, but also from a caloric viewpoint. In such respect, we remark that the prevailing group at $a=a_{2}^{*}$ is given by agents of type $\beta$, who assume a lower quantity of calories. Moreover, $K_{\beta}$ is slightly closer than $K_{\alpha}$ to the desirable calorie intake $\widehat{K}=2$, which allows maximizing the growth rate.

For the sake of completeness, we also investigate what happens to the calorie intakes at the other two locally stable equilibria in Figure 1 (F), i.e., at $a=0$ and at $a=1$. In such framework at $a=0$ we have $K_{\alpha}=13.151, K_{\beta}=1.010$ and at $a=1$ we have $K_{\alpha}=3.135, K_{\beta}=5.590$. Hence, at $a=0$ and at $a=1$ the calorie intake of the only surviving group is the closest to $\widehat{K}=2$, even if at $a=0$ we observe for the prevailing group a calorie intake lower than $\widehat{K}$, while at $a=1$ we observe for both groups an excess calorie intake. Actually, this is true not only at $a=0$ and at $a=1$, but also along the trajectories tending towards them.

We conclude our analysis by observing that in Figure 1, when increasing

\footnotetext{
${ }^{2}$ For the parameter configuration reported in Footnote 3 the unique stable equilibrium is given by $a=1$.

${ }^{3}$ We stress that we may obtain a nontrivial locally stable equilibrium even when the endowments of the two goods are both favorable to one group, as happens in Figure 1 (B) with agents of type $\beta$. This means that it is not true that the group with larger endowments, also for the commodity its agents have a stronger preference for, necessarily prevails, because of agents' pressure on the price formation mechanism. Indeed, even if the endowment of a commodity is larger with respect to the endowment of the other good, its price raises if the demand for it is too high. Due to the increased price, the agents who prefer it start consuming also the other commodity and this induces a decrease in their payoff, so that the share of the agents belonging to the other group grows and in such way no group disappears from the economy.
} 
the value of $w_{y, \alpha}$, we find two different kinds of bifurcations for the map $g$ : a transcritical bifurcation at $a=a^{*}=a_{2}^{*}=0.6425$ for $w_{y, \alpha}=1.5005$ and a saddle-node bifurcation at $a=a_{1}^{*}=a_{2}^{*}=0.3341$ for $w_{y, \alpha}=2.0846$ (see Figure $1(\mathrm{E})$ and $(\mathrm{G})$, respectively). Before stating in a formal manner the corresponding result in Proposition 5.1, we recall the main features related to the occurrence of such bifurcations. At a transcritical bifurcation a stable and an unstable equilibria merge and after that the stable equilibrium becomes unstable and vice versa. This is what happens to $a^{*}$ and $a_{2}^{*}$ in Figure 1: indeed for $w_{y, \alpha}$ in a left neighborhood of $1.5005 a^{*}$ is stable and $a_{2}^{*}$ unstable (cf. Figure 1 (D)), while for $w_{y, \alpha}$ in a right neighborhood of 1.5005 $a^{*}$ becomes unstable and $a_{2}^{*}$ gains stability (see Figure $1(\mathrm{~F})$ ), leading to the most interesting scenario analyzed above. At a (subcritical) saddle-node bifurcation a pair of hyperbolic equilibria, one stable and one unstable, coalesce at the bifurcation point, annihilate each other and disappear. This is what happens to $a_{1}^{*}$ and $a_{2}^{*}$ : namely, for $w_{y, \alpha}$ in a left neighborhood of $2.0846 a_{1}^{*}$ is unstable and $a_{2}^{*}$ stable (see Figure $1(\mathrm{~F})$ ), while for $w_{y, \alpha}$ in a right neighborhood of $2.0846 a_{1}^{*}$ and $a_{2}^{*}$ disappear, becoming complex (see Figure $1(\mathrm{H}))$.

Proposition 5.1 For the map $g=g\left(a ; w_{y, \alpha}\right)$ in (5.1) a transcritical bifurcation occurs at $\tilde{a}=0.6425$ for $\widetilde{w_{y, \alpha}}=1.5005$, while a saddle-node bifurcation occurs at $\tilde{a}=0.3341$ for $\widetilde{w_{y, \alpha}}=2.0846$.

Proof. According to [16, page 369], for the occurrence of a transcritical bifurcation we just have to check the following conditions:

$$
\begin{array}{ll}
g\left(\tilde{a} ; \widetilde{w_{y, \alpha}}\right)=0, & \frac{\partial g}{\partial a}\left(\tilde{a} ; \widetilde{w_{y, \alpha}}\right)=0, \quad \frac{\partial g}{\partial w_{y, \alpha}}\left(\tilde{a} ; \widetilde{w_{y, \alpha}}\right)=0, \\
\frac{\partial^{2} g}{\partial a \partial w_{y, \alpha}}\left(\tilde{a} ; \widetilde{w_{y, \alpha}}\right) \neq 0, & \frac{\partial^{2} g}{\partial a^{2}}\left(\tilde{a} ; \widetilde{w_{y, \alpha}}\right) \neq 0,
\end{array}
$$

while for the saddle-node bifurcation, we have to check the following conditions (cf. [16, page 364]):

$$
g\left(\tilde{a} ; \widetilde{w_{y, \alpha}}\right)=0, \quad \frac{\partial g}{\partial a}\left(\tilde{a} ; \widetilde{w_{y, \alpha}}\right)=0, \quad \frac{\partial g}{\partial w_{y, \alpha}}\left(\tilde{a} ; \widetilde{w_{y, \alpha}}\right) \neq 0, \quad \frac{\partial^{2} g}{\partial a^{2}}\left(\tilde{a} ; \widetilde{w_{y, \alpha}}\right) \neq 0 .
$$

Direct (software-assisted) computations show that all the above conditions are satisfied. In particular, it holds that:

$\partial^{2} g / \partial a \partial w_{y, \alpha}\left(\tilde{a} ; \widetilde{w_{y, \alpha}}\right)=1.559, \partial^{2} g / \partial a^{2}\left(\tilde{a} ; \widetilde{w_{y, \alpha}}\right)=6.4117, \partial g / \partial w_{y, \alpha}\left(\tilde{a} ; \widetilde{w_{y, \alpha}}\right)=$ -0.127 and $\partial^{2} g / \partial a^{2}\left(\tilde{a} ; \widetilde{w_{y, \alpha}}\right)=-3.9246$.

This completes the proof. 


\section{Conclusion}

In the present paper we added to the frameworks considered in $[1,2]$ the heterogeneity assumption for endowments, and we studied market equilibria, which link equilibrium price and optimal consumption quantities to population shares. The aim was indeed that of investigating whether the new heterogeneity hypothesis may generate multistability phenomena involving nontrivial market stationary equilibria with strong coexistence, at which the calorie intakes for the two groups of agents differ. We found that the answer is negative in the monotone population growth rate setting proposed in [1], while it is affirmative in the non-monotone growth rate framework in [2]. Finally, we performed a bifurcation analysis in order to understand how the market stationary equilibria with different calorie intakes do emerge. We obtained a qualitative result, showing that, according to the relative endowment values, those equilibria can appear through a transcritical or a saddle-node bifurcations. Our analysis allowed us to discuss the local stability and the basins of attraction of the stationary equilibria that emerge through such bifurcations.

We believe the settings considered, even in the simplified versions analyzed in $[1,2]$, can be a starting point for other research works.

From a mathematical viewpoint, it would indeed be interesting to study the various models taking time as discrete, rather than continuous, in order to investigate how the dynamics change and which new phenomena arise.

From a modeling viewpoint, the non-monotone frameworks analyzed here and in [2] could instead be modified to represent the fashion cycle. In such case, we would still deal with a bell-shaped map, describing, rather than the relationship between calorie intake and population growth rate, the link between consumption and imitative behavior, below the saturation level, and between consumption and snob behavior, above such level. In order to interpret the fashion cycle, and in particular its multistability phenomena, we need to identify (at least) two lifestyles, described by different preference structures; for each lifestyle we would introduce an attractiveness degree, which depends in a nonlinear bell-shaped manner on the consumption of the representative agent belonging to the population share who adopts that particular preference structure. Then, the two attractiveness degrees would jointly determine the population switching mechanism between the different lifestyles. 
This research did not receive any specific grant from funding agencies in the public, commercial, or not-for-profit sectors.

\section{References}

[1] Chang J, Stauber R. Evolution of preferences in an exchange economy. Econ Lett 2009;103:131-4.

[2] Naimzada A, Pireddu M. Endogenous evolution of heterogeneous consumers preferences: multistability and coexistence between groups. Econ Lett 2016;142:22-6.

[3] Ponthiere G. Existence and stability of overconsumption equilibria. Econ Modell 2011;28:74-90.

[4] Solomon CG, Manson JE. Obesity and mortality: a review of the epidemiological data. Am J Clin Nutr 1997;66:1044S-50S.

[5] Bender R, Trautner C, Spraul M, Berger M. Assessment of excess mortality in obesity. Am J Epidemiol 1998;147:42-8.

[6] Fontaine KR, Redden DT, Wang C, Westfall AO, Allison DB. Years of life lost due to obesity. J Am Med Assoc 2003;289:187-93.

[7] Adams KF, Schatzkin A, Harris TB, Kipnis V, Mouw T, Ballard-Barbash $\mathrm{R}$ et al. Overweight, obesity, and mortality in a large prospective cohort of persons 50 to 71 years old. New Engl J Med 2006;355:763-78.

[8] Waaler HT. Height, weight and mortality. The Norwegian experience. Acta Med Scand 1984;215,Suppl.679:1-56.

[9] Fogel RW. Economic growth, population theory and physiology: the bearing of long-term processes on the making of economic policy. Am Econ Rev 1994;84:369-95.

[10] Philipson TJ, Posner RA. Is the obesity epidemic a public health problem? A review of Zoltan J Acs and Alan Lyles's obesity, business and public policy. J Econ Lit 2008;46:974-82.

[11] Dindo P. A tractable evolutionary model for the Minority Game with asymmetric payoffs. Physica A 2005;355:110-8. 
[12] Zhao J, Szilagyi MN, Szidarovszky F. An $n$-person battle of sexes game. Physica A 2008;387:3669-77.

[13] Zhao J, Szilagyi MN, Szidarovszky F. n-person Battle of sexes game-a simulation study. Physica A 2008;387:3678-88.

[14] Cavalli F, Naimzada A, Pireddu M. A family of models for Schelling binary choices. Physica A 2016;444:276-96.

[15] Arthur WB. Increasing returns and path dependence in the economy. Ann Arbor: University of Michigan Press; 1994.

[16] Wiggins S. Introduction to applied nonlinear dynamical systems and chaos. Texts in applied mathematics. 2nd ed. New York: Springer; 2003. 